Article

\title{
Amoxicillin-Clavulanic Acid Empirical Oral Therapy for the Management of Children with Acute Haematogenous Osteomyelitis
}

\author{
Elena Serrano, Irene Ferri, Luisa Galli $\odot$ and Elena Chiappini * \\ Paediatric Infectious Disease Unit, Meyer Children's University Hospital, Department of Health Sciences, \\ University of Florence, Viale G.Pieraccini 24, 50139 Florence, Italy; elenaserrano0902@gmail.com (E.S.); \\ irene.ferri@stud.unifi.it (I.F.); luisa.galli@unifi.it (L.G.) \\ * Correspondence: elena.chiappini@unifi.it
}

Received: 27 May 2020; Accepted: 17 August 2020; Published: 18 August 2020

\begin{abstract}
According to the Guidelines of the European Society of Pediatric Infectious Diseases (ESPID), in low methicillin-resistant Staphylococcus aureus (MRSA) prevalence settings, short intravenous therapy is recommended in uncomplicated cases of acute haematogenous osteomyelitis (AHOM), followed by empirical oral therapy, preferentially with first/second-generation cephalosporin or dicloxacillin or flucloxacillin. However, several practical issues may arise using some of the first-line antibiotics such as poor palatability or adherence problems. Clinical, laboratory and therapeutic data from children with AHOM hospitalized in one Italian Paediatric Hospital between 2010 and 2019 were retrospectively collected and analyzed. The aim of the study was to highlight the extent of the use and the possible role of amoxicillin-clavulanic acid in the oral treatment of children with AHOM. Two hundred and ten children were included. S.aureus was identified in $42 / 58$ children $(72.4 \%$ of identified bacteria); $2 / 42$ S.aureus isolates were MRSA (4.8\%). No Kingella kingae was identified. Amoxicillin-clavulanic acid was the most commonly used oral drug $(60.1 \% ; n=107 / 178)$ and it was associated with clinical cure in all treated children. Overall, four children developed sequelae. One $(0.9 \%)$ sequela occurred among the 107 children treated with amoxicillin-clavulanic acid. Our results suggest that amoxicillin-clavulanic acid might be an option for oral antibiotic therapy in children with AHOM.
\end{abstract}

Keywords: acute haematogenous osteomyelitis; children; amoxicillin-clavulanic acid

\section{Introduction}

Acute haematogenous osteomyelitis (AHOM) is the most common musculoskeletal infection in children [1]. In most cases, AHOM is the consequence of hematogenous diffusion of a bacterial pathogen, commonly occurring in children under five years of age and in males [2]. Other risk factors for AHOM include history of recent trauma, recent febrile episodes or upper respiratory tract infections, prematurity, congenital or acquired immunodeficiency, or sickle cell disease [3]. Staphylococcus aureus is the most commonly isolated pathogen, accounting for 70-90\% of AHOM culture-positive cases [4]. Other microorganisms include Group-A Streptococcus pyogenes, Streptococcus pneumoniae, and Gram-negative rods [5]. Kingella kingae is an emergent pathogen, particularly in children aged $<4$ years [6]. Community-acquired methicillin-resistant $S$. aureus (MRSA) infections are increasingly reported in the United States, while low rates are observed in most European countries [7,8]. Moreover, an increasing frequency of Panton-Valentine leukocidin (PVL)-producing S. aureus isolates is reported $[9,10]$. Since microbiological diagnosis is obtained in about one-third of children, most cases undergo an empirical antibiotic therapy, based on individual clinical features, age, 
and local epidemiological data. In low/intermediate MRSA prevalence settings, antistaphylococcal penicillin (i.e., oxacillin or flucloxacillin), a cephalosporin, or clindamycin are the recommended first-line treatment. Historically, AHOM was treated with intravenous (IV) antibiotics for several weeks [1]. However, accumulating data support the use of short IV therapy followed by oral antibiotic therapy in uncomplicated cases [11-15] since this regimen appears to be as effective as longer antibiotic IV courses with no increased risk of complications or sequelae. Short IV therapy (about seven days) is now adopted by several centers in Europe [14] and the US [15]. The European Society of Paediatric Infectious Diseases (ESPID) guidelines for Bone and Joint infections [1] recommend switching from cefazolin to cephalexin or cefuroxime, IV clindamycin to oral clindamycin, and IV ceftriaxone + oxacillin to oral equivalents, such as dicloxacillin or flucloxacillin. At the same time, amoxicillin-clavulanic acid is considered an alternative option. However, "thorough evidence is lacking, and the tolerance is worse" [1,13-15]. However, several practical issues may arise. Some antibiotics considered as the first-choice drug by the ESPID guidelines may have low bone penetration (i.e., cephalexin, cefadroxil), poor palatability, adherence issues with four daily administrations (i.e., dicloxacillin, flucloxacillin), or poor tolerability (i.e., risk of a severe rash or Clostridium difficile diarrhea using clindamycin) [16-18]. Amoxicillin-clavulanic acid therapy would be more feasible in children. However, only a few studies are available in children with AHOM [4,5,19], and bone penetration varies from 10 to 20\% [20-22]. The aim of our study was to evaluate the management and outcome of AHOM in a third-level Italian university hospital over ten years, with particular consideration to the possible role of amoxicillin-clavulanic acid in the empirical oral therapy in children with AHOM.

\section{Results}

Overall, 210 children were included in the study (Table 1).

Table 1. Characteristics of the 210 study children.

\begin{tabular}{|c|c|c|}
\hline \multirow{2}{*}{$\operatorname{Sex}(n, \%)$} & Female & $83 / 210(39.52 \%)$ \\
\hline & Male & $127 / 210(60.48 \%)$ \\
\hline \multicolumn{2}{|c|}{ Median age (years; IQR) } & $5.5(2-11)$ \\
\hline \multicolumn{2}{|c|}{ Biopsy executed $(n, \%)$} & $33 / 210(15.71 \%)$ \\
\hline \multicolumn{2}{|c|}{ Positive blood PCR $(n, \%)$} & $8 / 119(6.72 \%)$ \\
\hline \multicolumn{2}{|c|}{ Positive pus/biopsy PCR $(n, \%)$} & $30 / 53(56.60 \%)$ \\
\hline \multicolumn{2}{|c|}{ Positive blood culture $(n, \%)$} & $36 / 85(42.35 \%)$ \\
\hline \multicolumn{2}{|c|}{ Positive pus/biopsy culture $(n, \%)$} & $23 / 45(51.11 \%)$ \\
\hline \multicolumn{2}{|c|}{ Staphylococcus aureus infection $(n, \%)$} & $42 / 210(20.00 \%)$ \\
\hline \multicolumn{2}{|c|}{ IV therapy; days (median, IQR) } & $18(10-23)$ \\
\hline \multicolumn{2}{|c|}{ IV therapy $<7$ days $(n ; \%)$} & $28 / 210(13.33 \%)$ \\
\hline \multicolumn{2}{|c|}{ IV therapy $\geq 7$ days $(n ; \%)$} & $182 / 210(86.67 \%)$ \\
\hline \multicolumn{2}{|c|}{ Oral therapy; days (median, IQR) } & $16(13-32)$ \\
\hline \multicolumn{2}{|c|}{ Total therapy; days (median, IQR) } & $41(28-58)$ \\
\hline \multirow{2}{*}{ IV drugs } & Single therapy & $9 / 210(9.05 \%)$ \\
\hline & Combination therapy & $191 / 210(90.95 \%)$ \\
\hline \multirow{2}{*}{ Oral therapy } & Single therapy & $130 / 210(61.90 \%)$ \\
\hline & Combination therapy & $45 / 210(21.43 \%)$ \\
\hline \multicolumn{2}{|l|}{ No oral switch } & $35 / 210(16.67 \%)$ \\
\hline \multirow{9}{*}{ Complicated AHOM } & Arthritis & $46 / 210(21.90 \%)$ \\
\hline & Sub-periosteal abscess & $35 / 210(16.67 \%)$ \\
\hline & Cellulitis & $29 / 210(13.81 \%)$ \\
\hline & Sepsis or septic shock & $23 / 210(10.95 \%)$ \\
\hline & Muscle abscess & $26 / 210(12.38 \%)$ \\
\hline & Pathologic fracture & $15 / 210(7.14 \%)$ \\
\hline & Intensive care unit & $10 / 210(4.76 \%)$ \\
\hline & Deep venous thrombosis & $5 / 210(2.38 \%)$ \\
\hline & Septic emboli & $2 / 210(1.090 .95 \%)$ \\
\hline
\end{tabular}

Note. IV: intravenous; IQR: interquartile range. 
Median age was 5.5 years. The lower limb was the most frequently affected site $(120 / 210 ; 57.14 \%)$, and the most commonly bones involved were femur (40/210; 19.05\%) and tibia (35/210; 16.67\%). Fever at onset was observed in $54.29 \%(n=114)$ cases. Other frequently observed symptoms were (pain 184/210; 87.62\%; functional limitation: 157/011; 74.76\%; swelling: 115/210; 54.8\%; rubor: 102/210; 48.57\%; erythema: $71 / 210 ; 33.8 \%$ ).

One hundred-sixteen out of 210 children (55.24\%) developed a complicated AHOM (Table 1). In particular 73/116 children presented with one complication (62.93\%), 26 children two complications $(22.41 \%)$ and 17 children developed at least three complications (14.66\%): specifically, $46 / 210$ (21.90\%) arthritis, 35/210 (16.8 16.67\%) sub-periosteal abscess, 29/210 (13.81\%) cellulitis, 23/210 (10.95\%) sepsis or septic shock, 26/210 (12.38\%) muscular abscess, $15 / 210$ (7.14\%) pathological fracture, $10 / 210(4.76 \%)$ required admission to an intensive care unit, 5/210 (2.38\%) children developed deep vein thrombosis and 2/210 (0.95\%) septic emboli.

\subsection{Inflammatory Indices}

Sixty-three point zero percent $(n=131 / 205)$ of children displayed white blood counts $(\mathrm{WBC})<12,000 / \mu \mathrm{L}$, and C-reactive protein $(\mathrm{CRP})<10 \mathrm{mg} / \mathrm{dL}$ in $84.782 .69 \%(n=150172 / 177208)$ of children CRP $<1 \mathrm{mg} / \mathrm{dL} 28.36 \%$ ( $n=59 / 208)$. Erythrocyte sedimentation rate (ESR) was $>20 \mathrm{~mm} / \mathrm{h}$ in $76.976 .61 \%(n=113131 / 147171)$ of cases.

\subsection{Microbiological Tests}

Overall, 153/210 (72.86\%) children underwent at least one microbiological test (Table 2). At least one organism was isolated in $37.9 \%$ (58/153) cases. S. aureus was the most commonly identified pathogen $(72.4 \%$ of identified bacteria, $42 / 58) ; 5.7 \%$ strains $(n=2)$ were Panton-Valentine leukocidin (PVL) producer, 2/42 S.aureus isolates were MRSA (4.8\%). No Kingella kingae strain was identified.

Table 2. Isolates in at least one microbiological test.

\begin{tabular}{cccccc}
\hline Organism & $\begin{array}{c}\text { Number of Isolated } \\
\text { Organisms }\end{array}$ & $\begin{array}{c}\text { Positive Blood } \\
\text { Culture }(\boldsymbol{n} ; \boldsymbol{\%})\end{array}$ & $\begin{array}{c}\text { Positive Pus/Biopsy } \\
\text { Culture }(\boldsymbol{n} ; \boldsymbol{\%})\end{array}$ & $\begin{array}{c}\text { Positive Blood } \\
\text { PCR }(\boldsymbol{n} ; \boldsymbol{\%})\end{array}$ & $\begin{array}{c}\text { Positive Pus/Biopsy } \\
\text { PCR }(\boldsymbol{n} ; \boldsymbol{\%})\end{array}$ \\
\hline Staphylococcus aureus & 42 & $21(72.41 \%)$ & $14(77.78 \%)$ & $1(33.33 \%)$ & $20(80.00 \%)$ \\
Streptococcus pyogenes & 7 & $2(5.56 \%)$ & $3(13.04 \%)$ & $1(12.5 \%)$ & $4(13.33 \%)$ \\
Proteus mirabilis & 2 & $0(0.00 \%)$ & $2(8.70 \%)$ & $0(0.00 \%)$ & $0(0.00 \%)$ \\
Pseudomonas aeruginosa & 2 & $0(0.00 \%)$ & $12(5.568 .70 \%)$ & $0(0.00 \%)$ & $1(3.33 \%)$ \\
Streptococcus agalactiae & 2 & $1(2.78 \%)$ & $0(0.00 \%)$ & $1(33.3312 .5 \%)$ & $0(0.00 \%)$ \\
Fusobacterium necrophorum & 1 & $0(0.00 \%)$ & $0(0.00 \%)$ & $0(0.00 \%)$ & $1(3.33 \%)$ \\
Streptococcus pneumoniae & 1 & $0(0.00 \%)$ & $0(0.00 \%)$ & $0(0.00 \%)$ & $1(3.33 \%)$ \\
Staphylococcus epidermidis & 2 & $2(5.56 \%)$ & $0(0.00 \%)$ & $0(0.00 \%)$ & $0(0.00 \%)$ \\
Escherichia coli & 1 & $0(0.00 \%)$ & $1(4.35 \%)$ & $0(0.00 \%)$ & $0(0.00 \%)$ \\
Staphylococcus hominis & 1 & $1(2.78 \%)$ & $0(0.00 \%)$ & $0(0.00 \%)$ & $0(0.00 \%)$ \\
Veilonella parvula & 1 & $1(2.78 \%)$ & $0(0.00 \%)$ & $0.00 \%)$ \\
Streptococcus mitis & 1 & $1(3.45 \%)$ & $0(0.00 \%)$ & $0(0.00 \%)$ & $0(0.00 \%)$ \\
Staphylococcus simulans & 1 & $0(0.00 \%)$ & $1(4.35 \%)$ & $0(0.00 \%)$ & $0.00 \%)$ \\
Corynebacterium amycolatum & 1 & $0(0.00 \%)$ & $1(4.35 \%)$ & $0(0.00 \%)$ & $0(0.00 \%)$ \\
\hline
\end{tabular}

Blood culture was performed in 85/210 (40.5\%) children and yielded positive results in 36/85 $(42.4 \%)$ cases. Culture on pus/biopsy was performed in 45/210 (21.4\%) cases; one pathogen was isolated in $18 / 45(40.0 \%)$ cases. PCR assay on a blood sample was performed in 119/210 (56.7\%) cases and was positive in 8/119 (6.7\%) children. PCR assay on pus/biopsy was performed in 53/210 $(25.2 \%)$ children and was positive in 30/53 (56.6\%) children. A positive result both in PCR and culture assays were obtained very few patients. In particular, in 28 children, both culture and PCR were positive and consistent ( $24 \mathrm{~S}$. aureus positive), in 25 children, culture was positive but PCR was negative (21 $S$ aureus positive), and, in five, culture was negative and PCR was positive ( $2 S$ aureus positive results)". 


\subsection{Imaging Studies}

Conventional radiography was performed in 185/210 children (88.1\%). A radiographic image compatible with AHOM was obtained in 76/185 (41.1\%) children. Magnetic resonance imaging was performed in 197/210 cases (93.8\%), and images compatible with a diagnosis of AHOM were obtained in 196/197 cases (99.5\%). Computed tomography scan was performed in 16/210 children (7.6\%), and images compatible with AHOM diagnosis were observed in 14/16 cases (87.5\%). Bone scintigraphy was performed in $8 / 210$ children $(3.8 \%)$, and results compatible with the diagnosis of osteomyelitis were detected in all cases.

\subsection{Antibiotic Therapy}

All 210 children received IV therapy. Median duration of IV therapy was 18 days (Table 1 ). The preferred IV regimen was oxacillin plus third generation cephalosporin $(n=130 / 210 ; 61.9 \%)$, followed by therapy including a glycopeptide $(n=22 / 210$ cases; $10.5 \%)$, or clindamycin ( $n=19 / 210$ cases; $9.0 \%)$, or clindamycin and teicoplanin $(n=2 / 210 ; 1.0 \%)$, or oxacillin in monotherapy $(n=11 / 210 ; 4.2 \%)$. Other used IV regimens were cefazolin $(n=4 / 210 ; 1.9 \%)$, linezolid $(n=3 / 210$; $1.4 \%)$, ceftazidime $(n=6 / 210 ; 2.9 \%)$, ampicillin/sulbactam $(n=7 / 210 ; 3.3 \%)$, amoxicillin-clavulanic acid ( $n=2 / 210 ; 1.0 \%)$, ceftazidime plus rifampicin ( $n=2 / 210 ; 1.0 \%)$, other regimens $(n=2 ; 1.0 \%)$

In 112/210 (53.3\%) children first-line IV therapy was switched to a second-line IV therapy for the following reasons: clinical failure $(n=17 / 210 ; 8.1 \%)$, bacterial identification $(n=23 / 210 ; 11.0 \%)$, adverse event ( $n=8 / 210 ; 3.8 \%)$, simplification $(n=35 / 210 ; 16.7 \%)$, antibiotic no longer available in hospital during treatment $(n=5 / 210 ; 2.4 \%)$. In $24 / 210$ cases $(11.4 \%)$, the reason was not specified. One hundred and seventy-eight children were switched to oral antibiotic therapy $(84.7 \%)$. The median duration of oral therapy was 16 days (Table 1 ).

Amoxicillin-clavulanic acid was the most commonly used oral drug $(n=107 / 178,60.1 \%)$. Other antibiotics used in oral therapy were co-trimoxazole plus rifampicin $(n=17 / 178 ; 9.6 \%)$, clindamycin $(n=11 / 178 ; 6.2 \%)$, and flucloxacillin $(n=6 / 178 ; 3.4 \%)$, cephalexin $(n=3 / 178 ; 1.7 \%)$, linezolid $(n=12 / 178 ; 6.7 \%)$, rifampicin + linezolid $(n=2 / 178 ; 1.1 \%)$, cefixime $(n=2 / 178 ; 1.1 \%)$, cefpodoxime $(n=2 / 178 ; 1.1 \%)$, clindamycin + rifampicin $(n=2 / 178 ; 1.1 \%)$, clindamycin + co-trimoxazole $(n=2 / 178 ; 1.1 \%)$, other regimens $(n=9 / 178 ; 5.1 \%)$. Thirty-nine out 210 children $(18.6 \%)$ underwent surgery.

\subsection{Follow-Up Results}

Seventeen out of 210 children $(8.1 \%)$ were switched to second-line IV therapy for clinical failure, and all of them resolved after the second-line treatment. Treatment failure with the need of rehospitalization was observed in five children $(2.4 \%)$, and three of these children were treated with amoxicillin-clavulanic acid (3/107; 2.8\%). Four children $(1.9 \%)$ developed sequela including two children with angular deformity and two with limited articular movement. One $(0.9 \%)$ sequela occurred among the 107 children treated with amoxicillin-clavulanic acid.

\section{Discussion}

The present study is a continuation of a previous one published in 2017 [23] and confirm other epidemiological data present in literature [24]. As expected, the microbiological diagnosis was reached in about one third of children, and S.aureus was the most commonly identified pathogen, being about $70 \%$ of the total. Moreover, the prevalence of MRSA infection is confirmed to be low in our setting (only 2/42 S. aureus isolates). Prevalence of MRSA strains was in line with those previously reported in Europe. In the United States the incidence of community-acquired MRSA (CA-MRSA) is increased [25], but in Europe CA-MRSA is still uncommon and below 2\% [26]. The reason for the low number of positive blood PCR in comparison to the positive blood culture in our study is unclear and more studies are needed at this regard. 
The preferred IV antibiotic regimen was oxacillin plus third-generation cephalosporin, adopted in more than $60 \%$ of children, while the most commonly prescribed oral antibiotic was amoxicillin-clavulanic acid, which was prescribed in a similar rate $(60 \%)$. Our data demonstrate the extensive use of amoxicillin-clavulanic acid $(>60 \%)$. Probably physicians considered its good activity against methicillin-sensitive $S$. aureus (MSSA), pharmacokinetics/pharmacodynamics profile and the low severe event event rate $[16,27]$. Interestingly, among children treated with amoxicillin-clavulanic acid, only three out of 107 needed rehospitalization, and only one $(0.9 \%)$ sequela occurred. Similarly, in a recent Danish study, amoxicillin-clavulanic acid was administered to 82 children (42 children with osteomyelitis [OM] 40 children with SA) for three weeks after IV cefuroxime for at least three days [13]. Relapse was reported in two children ( $2 \%)$ with AHOM, and permanent sequelae were observed in two children (2\%) [13] (Table 3). In Filleron et al. study, 176 children were included in the retrospective study: 56 with OM, 95 with SA and 25 with both OM and SA. Oral therapy with amoxicillin-clavulanic acid was administered in 82/176 children (46.6\%) [14]. Sequelae were observed in two children (1\%) [14] (Table 3). Roul-Levy et al. retrospectively compared two groups of children treated for AHOM $(n=45)$ [19]. One group was treated with oral amoxicillin-clavulanic acid (without IV therapy), and the other group had received two to four days of IV therapy (Cefamandolo) followed by oral amoxicillin-clavulanic acid for four weeks. Treatment failure was observed in four children $(8.9 \%)$ (one in the oral group and three in the IV group) [19] (Table 3). Complexly, these results evidence a large use of amoxicillin-clavulanic acid in children with AHOM, although this regimen is not the first-choice therapeutic option for oral therapy, according to the international guideline recommendations. Apparently, this drug seems to be administered to young children more easily than other molecules recommended as first line therapy. Available data suggest good efficacy.

Our study has several limitations due to its retrospective nature. Moreover, $31 \%$ of children did not underwent any microbiological tests. Another limitation is that the prevalence of MRSA varies from region to region, and therefore the observed low prevalence may not be the same throughout the nation. Another limitation is that a PCR using specific primers for K. kingae with a higher sensitivity than the broad-range 16S rDNA real-time PCR used in this study was available only starting from 2019 (performed only in 10 children). In addition, K. kingae is a fastidious to grow bacterium. This may explain the fact that no K. kingae was identified in our dataset.

In conclusion, in our dataset amoxicillin-clavulanic acid was used in two-thirds of children and associated with clinical cure and no failure in all of them. This finding is confirmed in larger studies $[13,14,19]$, and suggests that amoxicillin-clavulanic acid might by recommended for oral antibiotic therapy in children with AHOM. 
Table 3. Recent studies on the management of children with osteomyelitis and use of amoxicillin-clavulanic acid (amoxiclav).

\begin{tabular}{|c|c|c|c|c|c|c|c|c|c|c|c|}
\hline Study & Total & OM & $\begin{array}{l}\text { Median Age } \\
\text { (Years) }\end{array}$ & IV Therapy & $\begin{array}{l}\text { Duration IV } \\
\text { Therapy (Days) }\end{array}$ & Oral Amoxiclav $(n, \%)$ & $\begin{array}{c}\text { Dosage } \\
\text { (mg/kg Per Day) }\end{array}$ & $\begin{array}{l}\text { Duration oral } \\
\text { Therapy (Days) }\end{array}$ & Sequelae $(n, \%)$ & Relapse $(n, \%)$ & $\begin{array}{c}\text { S. aureus } \\
(n, \%)\end{array}$ \\
\hline Our data & 210 & 210 & 5.5 & $\begin{array}{c}\text { Oxacillin }+3 \mathrm{rd} \\
\text { generation } \\
\text { cephalosporin }(n=130, \\
61.9 \%), \text { other } \\
(n=80,38.1 \%)\end{array}$ & 18 & 107/178 (60.1\%) & 80 & 16 & $\begin{array}{l}4(1.9 \%)(1 \text { in oral } \\
\text { amoxiclav group })\end{array}$ & $\begin{array}{l}5(2.4 \%)(3 \text { in oral } \\
\text { amoxiclav group) }\end{array}$ & $42(72.4 \%)$ \\
\hline Nielsen et al. 2019 [13] & 82 & 42 & 4.9 & Cefuroxime & $6(\mathrm{OM}), 4$ (SA) & $82 / 82(100 \%)$ & - & $19(\mathrm{OM}) 11(\mathrm{SA})$ & $2(2.4 \%)$ & $2(2.4 \%)$ & $23(51 \%)$ \\
\hline Filleron et al. 2019 [14] & 176 & 56 & 1.8 & $\begin{array}{l}\text { Cloxacillin or amoxiclav } \\
+/- \text { gentamicin }\end{array}$ & 4 & $82 / 176(46.6 \%)$ & - & 11 & $2(1.1 \%)$ & - & $30(41 \%)$ \\
\hline Roul-Levy et al. 2016 [19] & 45 & 45 & 1.6 & Cefamandole $(n=26)$ & $2-4$ (IV group) & $\begin{array}{c}45 / 45(100 \%) \text { ( } 19 \text { exclusive } \\
\text { oral therapy; } 262-4 \text { day } \\
\text { IV therapy before } \\
\text { oral switch) }\end{array}$ & 80 & & $\begin{array}{l}4(8.9 \%) \text { ( } 3 \text { in IV } \\
\text { group; } 1 \text { in oral } \\
\text { group) }\end{array}$ & - & - \\
\hline
\end{tabular}

IV: intravenous; OM: osteomyelitis; SA: septic arthritis; amoxiclav: amoxicillin-clavulanic acid. 


\section{Methods}

\subsection{Definitions}

AHOM was defined as any bone infection with a period between symptoms onset and diagnosis $<$ two weeks [28,29]. Osteomyelitis was diagnosed in the presence of clinical (fever, swelling, warmth, pain, movements limitations) and radiological signs compatible with AHOM with or without bacterial isolation [26].

AHOM was defined complicated in the presence of sepsis, septic shock, arthritis, cellulitis, sub-periosteal or muscle abscess, deep vein thrombosis [26] pathological fracture, septic emboli or hospitalization in the intensive care unit [30].

\subsection{Study Design and Population}

A retrospective study was conducted in a single tertiary center, evaluating data of all children aged between one month and 18 years referred to the Meyer Paediatric University Hospital, Florence, Italy, from 1 January 2010, to 31 December 2019, with discharge code with the diagnosis of osteomyelitis (ICD code 10 M86.00-86.99), following The World Health Organization International Classification of Disease (WHO ICD-10). Inclusion criteria were: diagnosis of AHOM, as defined above; age between one month and 18 years. Exclusion criteria were: age $\leq 30$ days, congenital or acquired immunodeficiency, underlying bone disease, carrier of prosthetic material, open fracture or surgery at the infection's site or in an adjacent area, isolated septic arthritis (SA) (with no evidence of osteomyelitis in the adjacent area), hospital-acquired infections. The study is a continuation of a previous study published in 2017 [23]. Clinical, laboratory and therapeutic data of children with AHOM were collected and analyzed, as previously described [23]. In particular, clinical specimens for cultural analysis were collected into transport vials and inoculated onto an array of culture media suitable for detection of bacteria, and fungi (Columbia blood agar, chocolate agar, Schaedler CNA agar, Shaedler KKV agar, and sabouraud dextrose agar) after an enrichment step at $35^{\circ} \mathrm{C}$ in thioglycollate broth and nutrient broth. Cultures were carried out at $35{ }^{\circ} \mathrm{C}$ for $48 \mathrm{~h}$ under aerobic (Columbia blood agar and sabouraud dextrose agar), $5 \% \mathrm{CO}_{2}$ enriched (chocolate agar) or anaerobic (Schaedler CNA agar and Shaedler KKV agar) conditions. Identification of microbial isolates and antimicrobial susceptibility testing were carried out by the Vitek2 automated system (bioMérieux, Craponne, France).

Broad-range $16 S$ rDNA real-time PCR followed by PCR product sequencing was performed as previously prescribed [23]. Bacterial genomic DNA was extracted from $200 \mu \mathrm{L}$ of biological samples using the QIAmp DNA Easy Blood \& Tissue kit (Qiagen, Venlo, The Netherlands), according to the manufacturer's instructions. RT-PCR for several bacteria (Staphylococcus aureus, Streptococcus pyogenes, Pseudomonas aeruginosa, Kingella kingae) were performed using specific primers and probes, as previously described [23]. All reactions were performed in triplicates. A negative control (no template) and a positive control for each pathogen were included in every run. DNA was amplified in an ABI 7500 sequence detection system (Applied Biosystems, Foster City, CA, USA, brand of ThermoFisher, Waltham, MA, USA) using the following cycling parameters: $95{ }^{\circ} \mathrm{C}$ for $10 \mathrm{~min}$ followed by 45 cycles of a two-stage temperature profile of $95^{\circ} \mathrm{C}$ for $15 \mathrm{~s}$ and $60{ }^{\circ} \mathrm{C}$ for $1 \mathrm{~min}$.

Author Contributions: Conceptualization, E.C. and E.S.; Data Curation, E.C., I.F., and E.S.; Methodology, L.G.; Formal Analysis, E.S., I.F., and E.C.; Investigation, E.S.; Supervision, E.C. and L.G.; Validation, E.C., L.G. and E.S.; Writing-Original Draft Preparation, E.S., L.G., E.C.; Writing - Review \& Editing, L.G. All authors have read and agreed to the published version of the manuscript.

Funding: The authors have no funding to disclose.

Conflicts of Interest: The authors have no conflict of interest to disclose. 


\section{References}

1. Saavedra, J.; Falup-Pecurariu, O.; Faust, S.; Girschick, H.; Hartwig, N.; Kaplan, S.; Lorrot, M.; Mantadakis, E.; Peltola, H.; Rojo, P.; et al. Practice Guideline Bone and infections. 2017. Available online: http://links.lww. com/INF/C729 (accessed on 6 May 2020).

2. Whyte, N.S.B.; Bielski, R.J. Acute Hematogenous Osteomyelitis in Children. Rev. Pediatr Ann. 2016, 45, e204-e208. [CrossRef] [PubMed]

3. Gigante, A.; Coppa, V.; Marinelli, M.; Giampaolini, N.; Falcioni, D.; Specchia, N. Acute osteomyelitis and septic arthritis in children: A systematic review of systematic reviews. Eur. Rev. Med. Pharmacol. Sci. 2019, 23, 145-158. [PubMed]

4. Thomsen, I.; Creech, C.B. Advances in the diagnosis and management of pediatric osteomyelitis. Curr. Infect. Dis. Rep. 2011, 13, 451-460. [CrossRef]

5. Congedi, S.; Minotti, C.; Giaquinto, C.; Da Dalt, L.; Donà, D. Acute infectious osteomyelitis in children: New treatment strategies for an old enemy. World J. Pediatr. 2020. [CrossRef] [PubMed]

6. Yagupsky, P.; Porsch, E.; St Geme, J.W., 3rd. Kingella kingae: An emerging pathogen in young children. Pediatrics 2011, 127, 557-565. [CrossRef] [PubMed]

7. Sutter, D.E.; Milburn, E.; Chukwuma, U.; Dzialowy, N.; Maranich, A.M.; Hospenthal, D.R. Changing susceptibility of Staphylococcus aureus in a US pediatric population. Pediatrics 2016, 137, e20153099. [CrossRef]

8. Del Giudice, P.; Tattevin, P.; Etienne, J. Infections à Staphylococcus aureus résistants à la méticilline communautaires. Presse Med. 2012, 41, 713-720. [CrossRef]

9. Gonzalez, B.E.; Teruya, J.; Mahoney, D.H.; Hulten, K.G., Jr.; Edwards, R.; Lamberth, L.B.; Hammerman, W.A.; Mason, E.O.; Kaplan, S.L., Jr. Venous thrombosis associated with staphylococcal osteomyelitis in children. Pediatrics 2006, 117, 1673-1679. [CrossRef]

10. Kok, E.Y.; Vallejo, J.G.; Sommer, L.M.; Rosas, L.; Kaplan, S.L.; Hulten, K.G.; McNeil, J.C. Association of Vancomycin MIC and Molecular Characteristics with Clinical Outcomes in Methicillin-Susceptible Staphylococcus aureus Acute Hematogenous Osteoarticular Infections in Children. Antimicrob. Ag. Chemother. 2018, 62, e00084-18. [CrossRef]

11. Michelow, I.C.; Mandell, J.G. Sequential Intravenous Oral Antibiotic Therapy for Osteomyelitis: How Short Is Long Enough? JAMA Pediatr. 2015, 169, 698-699. [CrossRef]

12. Peltola, H.; Pääkkönen, M.; Kallio, P.; Kallio, M.J. Short-versus long-term antimicrobial treatment for acute hematogenous osteomyelitis of childhood: Prospective, randomized trial on 131 culture-positive cases. Pediatr. Infect. Dis. J. 2010, 29, 1123-1128. [CrossRef] [PubMed]

13. Nielsen, A.B.; Nygaard, U.; Hoffmann, T.; Kristensen, K. Short individualized treatment of bone and joint infections in Danish children. Arch. Dis. Child. 2019, 104, 205-206. [CrossRef] [PubMed]

14. Filleron, A.; Laurens, M.E.; Marin, G.; Marchandin, H.; Prodhomme, O.; Alkar, F.; Godreuil, S.; Nagot, N.; Cottalorda, J.; L'Kaissi, M.; et al. Short-course antibiotic treatment of bone and joint infections in children: A retrospective study at Montpellier University Hospital from 2009 to 2013. J. Antimicrob. Chemother. 2019, 74, 3579-3587. [CrossRef] [PubMed]

15. Howard-Jones, A.R.; Isaacs, D. Systematic review of duration and choice of systemic antibiotics therapy for acute haematogenous bacterial osteomyelitis in children. J. Paediatr. Child Health 2013, 49, 760-768. [CrossRef]

16. Chiappini, E.; Serrano, E.; Galli, L.; Villani, A.; Krzysztofiak, A. Italian Paediatric Collaborative Osteomyelitis Study Group. Practical Issues in Early Switching from Intravenous to Oral Antibiotic Therapy in Children with Uncomplicated Acute Hematogenous Osteomyelitis: Results from an Italian Survey. Int. J. Environ. Res. Public Health 2019, 16, 3557. [CrossRef]

17. Rouse, C.; Mistry, P.; Rayner, O.; Nickless, J.; Wan, M.; Southern, K.W.; Batchelor, H.K. A mixed-methods study of the administration of flucloxacillin oral liquid; identifying strategies to overcome administration issues of medicines with poor palatability. Int. J. Pharm. Pract. 2017, 25, 326-334. [CrossRef]

18. Gerding, D.N. Clindamycin, Cephalosporins, Fluoroquinolones, and Clostridium Difficile-Associated Diarrhea: This Is an Antimicrobial Resistance Problem. Clin. Infect. Dis. 2004, 38, 646-648. [CrossRef]

19. Roul-Levy, A.; Looten, V.; Bachy, M.; Grimprel, E.; Carbajal, R.; Vialle, R. Oral Ambulatory Treatment of Acute Osteomyelitis in Children: A Case-Control Study. Pediatr. Emerg. Care 2016, 32, 154-156. [CrossRef] 
20. Landersdorfer, C.B.; Bulitta, J.B.; Kinzig, M.; Holzgrabe, U.; Sörgel, F. Penetration of antibacterials into bone: Pharmacokinetic, pharmacodynamic and bioanalytical considerations. Clin. Pharmacokinet. 2009, 48, 89-124. [CrossRef]

21. Thabit, A.K.; Fatani, D.F.; Bamakhrama, M.S.; Barnawi, O.A.; Basudan, L.O.; Alhejaili, S.F. Antibiotic penetration into bone and joints: An updated review. Int. J. Infect. Dis. 2019, 81, 128-136. [CrossRef]

22. Boselli, E.; Allaouchiche, B. Diffusion in bone tissue of antibiotics. Presse Med. 1999, 28, 2265-2276. [PubMed]

23. Chiappini, E.; Camposampiero, C.; Lazzeri, S.; Indolfi, G.; De Martino, M.; Galli, L. Epidemiology and Management of Acute Haematogenous Osteomyelitis in a Tertiary Paediatric Center. Int. J. Environ. Res. Public Health 2017, 14, 477. [CrossRef] [PubMed]

24. Arnold, G.C.; Bradley, J.S. Osteoarticular infections in children. Infect. Dis. Clin. N. Am. 2015, 29, 557-574. [CrossRef] [PubMed]

25. Funk, S.S.; Copley, L.A. Acute Hematogenous Osteomyelitis in Children: Pathogenesis, Diagnosis, and Treatment. Orthop. Clin. N. Am. 2017, 48, 199-208. [CrossRef]

26. Calvo, C.; Nunez, E.; Camacho, M.; Clemete, D.; Fernandez-Cooke, E.; Alcobendas, R.; Mayol, L.; Soler-Palacin, P.; Oscoz, M.; Saavedra-Lozano, J. Epidemiology and management of acute, uncomplicated, septic arthritis and osteomyelitis. Pediatr. Infect. Dis. J. 2016, 35, 1288-1293. [CrossRef] [PubMed]

27. Gerber, J.S.; Ross, R.K.; Bryan, M.; Localio, A.R.; Szymczak, J.E.; Wasserman, R.; Barkman, D.; Odeniyi, F.; Conaboy, K.; Bell, L.; et al. Association of broad- vs narrow-spectrum antibiotics with treatment failure, adverse events, and quality of life in children with acute respiratory tract infections. JAMA 2017, 318, 2325-2336. [CrossRef] [PubMed]

28. Dartnell, J.; Ramachandran, M.; Katchburian, M. Haematogenous acute and subacute paediatric osteomyelitis. A systematic review of the literature. J. Bone Jt. Surg. 2012, 94, 584-595. [CrossRef]

29. Peltola, H.; Päkköönen, M. Acute Osteomyelitis in Children. N. Engl. J. Med. 2014, 370, 352-360. [CrossRef]

30. Chiappini, E.; Mastrangelo, G.; Lazzeri, S. A case of osteomyelitis: An update on diagnosis and treatment. Int. J. Environ. Res. Public Health 2016, 13, 539. [CrossRef]

(C) 2020 by the authors. Licensee MDPI, Basel, Switzerland. This article is an open access article distributed under the terms and conditions of the Creative Commons Attribution (CC BY) license (http://creativecommons.org/licenses/by/4.0/). 